\title{
Origin of electronic localization in metal- insulator transition of phase change materials
}

Cite as: Appl. Phys. Lett. 113, 263502 (2018); https://doi.org/10.1063/1.5053574

Submitted: 23 August 2018 . Accepted: 04 December 2018 . Published Online: 27 December 2018

I. Sarkar, K. Perumal, S. Kulkarni, and W. Drube (iD)

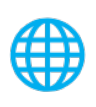

View Online

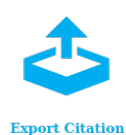

Export Citation

\section{ARTICLES YOU MAY BE INTERESTED IN}

Transport properties of near surface InAs two-dimensional heterostructures Applied Physics Letters 113, 262104 (2018); https://doi.org/10.1063/1.5050413

Strain-induced changes in AlGaN/GaN two-dimensional electron gas structures with low surface state densities

Applied Physics Letters 113, 263503 (2018); https://doi.org/10.1063/1.5079400

Probing spin-orbit interaction via Fano interference

Applied Physics Letters 113, 261104 (2018); https://doi.org/10.1063/1.5080697

\section{Applied Physics Reviews} Now accepting original research 


\title{
Origin of electronic localization in metal-insulator transition of phase change materials
}

\author{
I. Sarkar, ${ }^{1, a)}$ K. Perumal, ${ }^{2}$ S. Kulkarni, ${ }^{3}$ and W. Drube ${ }^{2}$ \\ ${ }^{1}$ Institute of Nano Science and Technology, Mohali, Punjab 160062, India \\ ${ }^{2}$ DESY Photon Science, Deutsches Elektronen-Synchrotron, 22603 Hamburg, Germany \\ ${ }^{3}$ Centre for Materials for Electronics Technology, Panchavati, Pune 411008, India
}

(Received 23 August 2018; accepted 4 December 2018; published online 27 December 2018)

Tellurium based phase change materials are unique 3D-solids proposed to undergo Anderson type metal-insulator transition. However, the origin of this transition is not unambiguously understood. Here, we report combined high energy resolution photoemission spectroscopy and high k-resolution X-ray diffraction measurements on a reversibly phase switched $\mathrm{Ge}_{2} \mathrm{Sb}_{2} \mathrm{Te}_{5}$ film. The results resolve the ambiguity between previous spectroscopic data and the proposed theoretical model for the origin of Anderson localization in these materials. Furthermore, by switching between the metallic state to insulating and back to metallic, we probe the electronic structure evolution in the phase change material. Published by AIP Publishing.

https://doi.org/10.1063/1.5053574

The concept of Anderson localization (AL) provides an excellent opportunity for controlling metal insulator transitions (MITs) by tuning disorder. ${ }^{1-3}$ But in most solids, fermionic excitations are responsible for electronic transport. Therefore, MIT can also be driven by electronic correlations, ${ }^{1-5}$ and it is hard to find $3 \mathrm{D}$ crystalline solids where it occurs through $\mathrm{AL}$ although it has been studied in optical lattices. $^{2,6-8}$ Recently, transport measurements have been reported $^{5}$ pointing towards AL-type MIT in 3D-crystalline phase change materials (PCMs) of chalcogenide alloys, comprising Germanium (Ge), Antimony (Sb), and Tellurium (Te), known as GST. Following this study, density functional theory (DFT) calculations of the electronic structure of GST near the Fermi energy $\left(\mathrm{E}_{F}\right)^{9}$ have been carried out to propose a possible origin of $\mathrm{AL}$ in PCM. Calculations suggest that MIT in GST is driven by localization of Te p-states by vacancies present. However, this model has not been confirmed experimentally by electronic structure studies and remains ambiguous in the light of earlier spectroscopic data which suggest no change in the initial Te-states between metallic and insulating phases. ${ }^{10}$

GST is an extremely important device material, and understanding the origin of its phase change properties holds key for the development of the next generation of PCM based devices. ${ }^{11,12}$ GST comprises many vacancies in the Ge and $\mathrm{Sb}$ sublattices that get introduced as quenched disorder during growth. ${ }^{13-15}$ The Te p-states contribute mainly to the occupied density of states (DOS) near $\mathrm{E}_{F}{ }^{9,16,17}$ DFT calculations show that an increased randomness of vacancy clusters surrounding the $\mathrm{Te}$ site causes localization of the p-state wave-functions near $\mathrm{E}_{F}$, leading to the insulating phase in GST. ${ }^{9}$ Vacancy ordering reduces the localization of the Te p-states, leading to a recovery of the metallic state. This was concluded by computing the variation of the Te p-state DOS near $\mathrm{E}_{F}$ as a function of vacancy ordering. ${ }^{9}$ However, earlier reports of photoelectron spectroscopy data on

a)indranil.sarkar@inst.ac.in poly-crystalline and amorphous GST suggest that the initial Te states are unchanged between crystalline and amorphous phases although the final state of Te photoemission peaks is affected by a strong change in the free electron carrier density. ${ }^{10}$ These data suggest that the change in the carrier concentration along with the change in order related to $\mathrm{Ge}$ and $\mathrm{Sb}$ co-ordination drives the MIT in GST. This is incongruent to the DFT calculations which require a change in the vacancy environment surrounding the Te sites for $\mathrm{p}$-state localization $^{9}$ which should affect the Te initial states. This ambiguity can be resolved by experimental data on the electronic structure of single crystalline and amorphized GST. It is also important to obtain spectroscopic data on recrystallized GST, which are so far missing in the reported electronic structure studies on polycrystalline and amorphous GST. ${ }^{10,16,18}$ This is important as GST can be switched ultrafastly between the metallic-crystalline and the insulatingamorphous phase by laser annealing. ${ }^{14,15,19,20}$ Hence, a spectroscopic study combining data from single crystalline, amorphized, and re-crystallized GST is required to obtain electronic structure information for an understanding of the MIT switching in GST.

To address the above issues, here we present the electronic structure study on single crystalline (c-), amorphized (a-), and re-crystallized (r-) GST using high energy resolution hard X-ray photoelectron spectroscopy (HAXPES) in combination with high k-resolution X-ray diffraction (XRD) to characterize the vacancy related structural order. The data were taken on the identical area of the same samples, which are crucial to unambiguously reveal the electronic structure evolution upon switching between the crystalline and amorphous states. The reversible switching was accomplished by ultrashort laser pulse annealing. The diffraction study is important as recent XRD studies on GST show ordering of vacancies to form a layer in the crystalline phase instead of being randomly distributed in the $\mathrm{Ge} / \mathrm{Sb}$ sublattice, as is the case in the amorphous phase. ${ }^{13}$ In the present work, the combined spectroscopic and diffraction data allow 
us to directly correlate the electronic structure change with vacancy layer ordering. The choice of HAXPES is well suited to measure the bulk electronic structure of $\mathrm{GST}^{18,21}$ and in particular is a better representative of variation in initial-state DOS near the Fermi level compared to low energy photoemission studies. ${ }^{22}$

The c-GST films were prepared on a $\mathrm{Si}(111)$ substrate using molecular beam epitaxy at a base pressure of $10^{-10}$ mbar. Thin films of $\approx 21 \mathrm{~nm}$ thickness were grown using the $\mathrm{Ge}_{2} \mathrm{Sb}_{2} \mathrm{Te}_{5}$ source material having a purity of $99.99 \%$ on the substrate maintained at $250^{\circ} \mathrm{C}$. To characterize the crystallinity of the samples, high-resolution XRD measurements were done at a photon energy of $25 \mathrm{keV}$ using synchrotron radiation (PETRA III beamline P08, DESY, Hamburg, Germany). The XRD data [Fig. 1(a)] show sharp peaks at $\mathrm{Q}_{z}$ $=2.00,4.01,6.01 \AA^{-1}$ originating from the Si substrate and three peaks at $\mathrm{Q}_{z}=1.8,3.6$, and $5.4 \AA^{-1}$ corresponding to the (111), (222), and (333) reflections of the GST epilayer. ${ }^{13}$ Additional broad features (VLp) are observed at $\mathrm{Q}_{z}=1.5$, 3.3, and $5.1 \AA^{-1}$. These are superstructure peaks appearing due to the vacancy layer formed by occupational modulation in the $\mathrm{Sb} / \mathrm{Ge}$ sublattice. ${ }^{13}$ The amorphization of $\mathrm{c}-\mathrm{GST}$ to a-GST and subsequent recrystallization to r-GST were carried out using light pulses from a $15 \mathrm{~W}$ high-power ultrafast laser (Pharos, TOPAG Lasertechnik $\mathrm{GmBH}$ ). Amorphization was done by a single pulse of $\approx 80 \mathrm{~mJ} / \mathrm{cm}^{2}$ fluence. The re-crystallization was accomplished using 400 pulses of $\approx 18 \mathrm{~mJ} / \mathrm{cm}^{2}$ fluence. Amorphization and
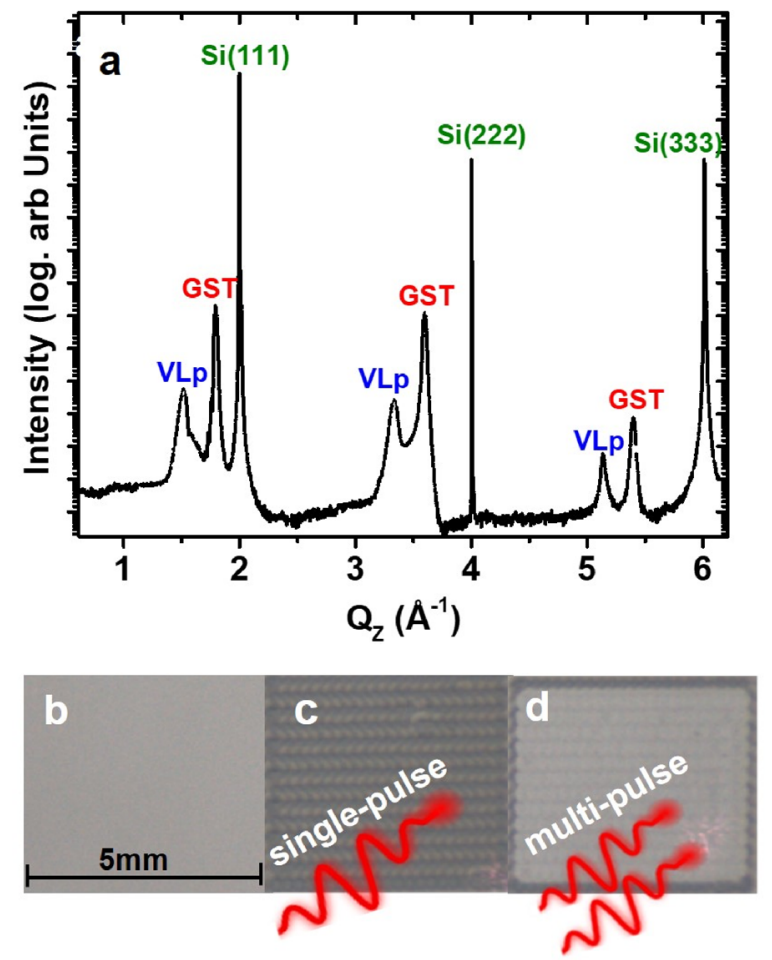

FIG. 1. (a) XRD data for GST grown on Si(111). Substrate, GST, and vacancy layer peaks (VLp) are indicated. (b)-(d) show optical images of crystalline c-GST (b), amorphized a-GST (c), and re-crystallized r-GST (d). A reduced optical reflectivity is observed for a-GST. The same area on the c-GST sample is chosen for single laser pulse amorphization and multipulse re-crystallization. The dark lines around the edge of the r-GST image correspond to the parts of a-GST, which have not been re-crystallized. The optical reflectivity is recovered in r-GST. re-crystallization were monitored using visible light reflectivity of the surface. Figures $1(\mathrm{~b})-1(\mathrm{~d})$ show the optical picture of c-GST (b), a-GST (c), and r-GST (d). As expected, a-GST exhibits a strong reduction in optical reflectivity with respect to c-GST. Upon re-crystallization, r-GST regains its optical reflectivity. The HAXPES measurements were done at $5.95 \mathrm{keV}$ X-ray excitation (PETRA III, beamline P09). ${ }^{23}$ The exciting $\mathrm{X}$-rays were monochromatized by a combination of a $\mathrm{Si}(111)$ double-crystal primary monochromator and a $\mathrm{Si}(333)$ channel-cut post-monochromator to attain a high energy resolution. $^{23}$

The HAXPES measurements were performed in such a way that crystalline, amorphous, and recrystallized regions having uniform optical contrast were measured. So, there was no influence in the spectroscopic data coming from the regions of non-uniform optical contrast at the outer fringes of the laser irradiated spot on the sample, which can have left over mixed areas of crystalline and amorphous nature. For HAXPES measurements, the X-ray beam size was $100 \mu \mathrm{m} \times 200 \mu \mathrm{m}$. The measurements were carried out in the small area acceptance mode that captured data from the region of interest of diameter $100 \mu \mathrm{m}$. This allowed recording of data purely from uniform crystalline or amorphous regions without capturing photo-emission signals from the outer fringes around the edges of the laser irradiated spot on the sample. Alignment of the uniform sample area of uniform contrast was carried out using a long focal distance microscope. The microscope allowed viewing through an iris behind the spectrometer along the path of electron optics. Figure 2(a) shows the GST valence band (VB) spectra, which can be divided into two regions. Region-1 between binding energies of $6.5 \mathrm{eV}$ and $1.5 \mathrm{eV}$ is very similar for c-, a-, and $\mathrm{r}-\mathrm{GST}$. At $\approx 1.5 \mathrm{eV}$, all spectra exhibit a peaked maximum, indicating a higher density of localized states. DFT calculations predict that this is ideal for wavefunction localization. ${ }^{9}$ This is corroborated by the fact that the a-GST spectra [Fig. 2(b)] sharply drop below $1.5 \mathrm{eV}$, opening an insulating gap of $\approx 0.3 \mathrm{eV}$ near $\mathrm{E}_{F}$.

However, it is interesting to find that there is a pronounced difference in the spectral weight in the region

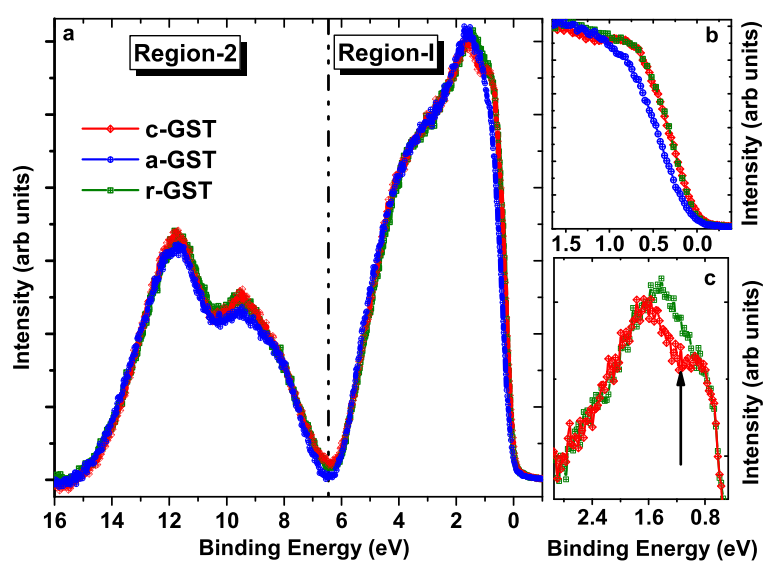

FIG. 2. (a) Valence band spectra $(h \nu=5.95 \mathrm{keV})$ for GST grown on $\mathrm{Si}(111)$. (b) Close-up of the valence band maximum, the bandgap opening for a-GST (blue curve) is clearly seen near $E_{F}$. (c) Comparison of c- and $r-$ GST showing the non-monotonic behavior of the spectral shape of c-GST ("dip") around $1.15 \mathrm{eV}$ (arrow) in contrast to r-GST which behaves monotonically. 
between the VB peak and $\mathrm{E}_{F}$ of the initial c-GST and final r-GST crystalline samples. While the c-GST spectrum exhibits a non-monotonic slope, the r-GST shows a monotonic decrease. As expected for the metallic state, both c-GST and r-GST do not show any gap at $\mathrm{E}_{F}$.

Around $1.15 \mathrm{eV}$, the c-GST spectrum exhibits a pronounced "dip" in its spectral weight [arrow in Fig. 2(c)] followed by an intensity rise around $0.93 \mathrm{eV}$. By contrast, the r-GST spectrum is monotonic in the same energy region towards $\mathrm{E}_{F}$. The spectral intensity of r-GST is larger compared to c-GST, suggesting a higher DOS in the metallic states [Fig. 2(c)]. A higher spectral intensity and the absence of a gap at $\mathrm{E}_{F}$ confirm that the observed effect is not due to any residual amorphous state in r-GST. The results show that the switching of localized states in insulating a-GST to delocalized states in metallic r-GST ends in a different DOS structure compared to that of the initial metallic c-GST. This suggests that reversible switching in the PCM evolves through different electronic paths. However, on recrystallizing the r-GST phase second time, the spectral behavior is not found to change with respect to the one observed in the r-GST phase (cf. supplementary material Fig. S1). The observed difference in c-GST and r-GST electronic structures is concomitant to the earlier report showing that the electrical resistivity of as-grown crystalline MBE films of GST is different from that of laser annealed crystallized films. ${ }^{13}$ The different intrinsic nature of the c-GST and $r$ GST metallic states is also confirmed by the Te $3 \mathrm{~d}$ core level and MNN Auger spectra, as discussed below. In VB region2 between binding energies of $6.5 \mathrm{eV}$ and $15 \mathrm{eV}$ [Fig. 2(a)], the c-GST and r-GST spectra are almost identical. However, the a-GST spectrum shows a slightly broadened spectral weight distribution. This has also been observed in previous studies and been attributed to enhanced disorder in the system. ${ }^{21}$ This region can be fitted assuming three components [Figs. 3(a) and 3(b)] corresponding to $\mathrm{Te} 5 \mathrm{~s}, \mathrm{Sb} 5 \mathrm{~s}$, and $\mathrm{Ge}$ $4 \mathrm{~s}$ bands. ${ }^{16,17,21}$ The composition obtained from the fit yields an atomic ratio of $\{2( \pm 0.1): 2( \pm 0.1): 5( \pm 0.1)\}$ for $\{\mathrm{Ge}: \mathrm{Sb}: \mathrm{Te}\}$, consistent with the nominal composition of $\mathrm{Ge}_{2} \mathrm{Sb}_{2} \mathrm{Te}_{5}$. The Gaussian (G) to Lorentzian (L) ratio for
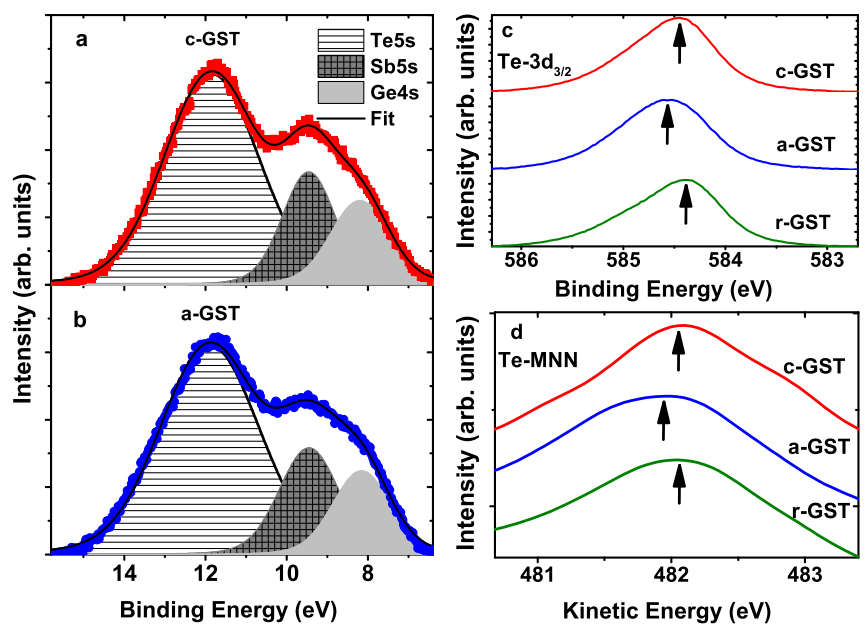

FIG. 3. Compositional analysis of valence band region-2 for (a) c-GST and (b) a-GST. (c) Te $3 d_{3 / 2}$ emission profile. (d) Te MNN Auger peak for c-, a-, and r-GST; the arrows mark the peak position.
$\{\mathrm{Te}: \mathrm{Sb}: \mathrm{Ge}\}$ is found to be $\{9.59: 5.28: 5.66\}$ for c-GST and $\{10.07: 5.88: 5.7\}$ for a-GST, respectively. There is a marginal increase in the G:L ratio for a-GST, which indicates higher static disorder in a-GST compared to the crystalline samples. ${ }^{21}$ As the region near $\mathrm{E}_{F}$ is derived from $\mathrm{Te} 4 \mathrm{p}$ states, ${ }^{9,16,17}$ it is not clear whether such a marginal change in static Te disorder can cause localization. However, the Te $3 \mathrm{~d}$ core level spectra of a-GST compared to c-GST show an $\approx 150 \mathrm{meV}$ shift towards higher binding energy [Fig. $3(\mathrm{c})$ ], which is consistent with the gap opening near $\left(\mathrm{E}_{F}\right)$. A similar shift is also observed for other core level spectra (cf. supplementary material Figs. S2 and S3). In a previous spectroscopic study report of a poly-crystalline sample, ${ }^{10}$ such a shift in the Te core level has been ascribed to a final state effect because of the change in the screening efficiency of the core hole due to the decrease in free carrier density in the amorphous phase. This was suggested based on the expectation that the core level shifts related to final state effects should show a three times larger energy shift of the Te MNN Auger emission compared to the Te $3 \mathrm{~d}$ peak. ${ }^{10}$ However, our measurements clearly show a Te MNN shift of $\approx 150 \mathrm{meV}$ [Fig. 3(d)] to lower kinetic (higher binding) energy in a-GST with respect to c-GST and r-GST, which is comparable to the observed corresponding shift of the Te $3 \mathrm{~d}$ core level. This indicates an initial state effect related to the change in the Te environment of a-GST with respect to c- and r-GST. This observation supports the theoretical model attributing the change in the vacancy cluster environment around the $\mathrm{Te}$ atom as the driving mechanism for the localization in the insulating state. ${ }^{9}$ The Te $3 \mathrm{~d}$ core level and Te MNN Auger peaks of r-GST shift $\approx 25 \mathrm{meV}$ to lower kinetic (higher binding) energy compared to c-GST, confirming the observed DOS behavior near $\mathrm{E}_{F}$ and pointing towards a different path for the delocalization of Te states on re-crystallization. In order to correlate the observed electronic structure changes with the extent of structural disorder, the XRD pattern in the $\mathrm{Si}(222)$ region (Fig. 4) of c-, a-, and r-GST along with the vacancy layer peak (shaded region) was measured with a high resolution. In each case, there is a GST diffraction peak around $3.6 \AA^{-1}$. Note that for c-GST, additional Laue oscillations are observed on both sides of the crystalline peak,

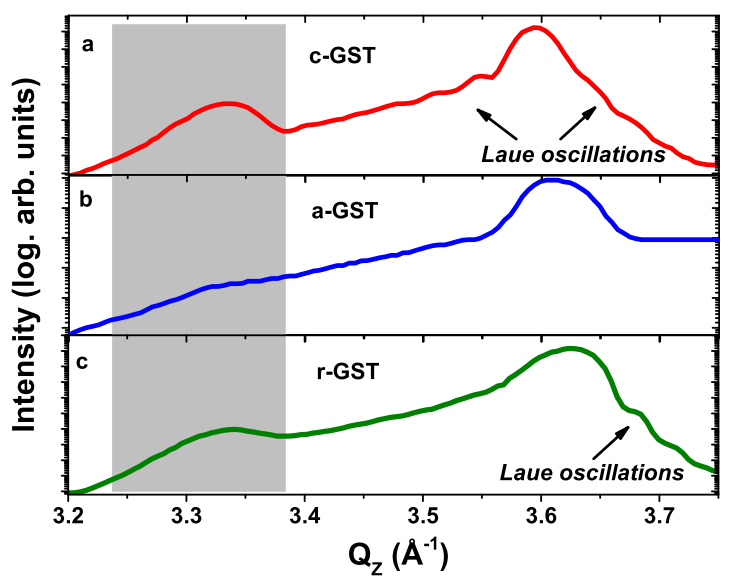

FIG. 4. XRD data of GST in the $\mathrm{Si}(222)$ peak region. The shaded regions indicate the vacancy layer. For c-GST and r-GST, Laue oscillations are visible, proving a high degree of crystallinity. 
indicating a high degree of crystallinity. These oscillations correspond to a crystalline thickness of $2 \pi / \Delta \mathrm{q} \approx 21 \mathrm{~nm}$. After amorphization, they are absent for a-GST but clearly reappear in r-GST, confirming re-crystallization of the sample. Interestingly, the amorphized sample still shows a weak GST peak implying some residual crystalline signals coming from the outer fringes of the amorphous region. These fringe areas do not affect the photoemission results as the data are collected from a region $(\sim 100 \mu \mathrm{m} \times 100 \mu \mathrm{m})$ which only covers the crystalline area and does not overlap with the fringe areas. However, the diffraction spot is larger and in order to reduce effects from fringes, more focussed beams are required.

Relative to c-GST, the GST peaks in r- and a-GST are slightly shifted towards higher $\mathrm{Q}_{z}$, indicating out-of-plane relaxation, in accord with previous reports. ${ }^{13}$ The broad structure (shaded region) fingerprinting the ordering of the vacancy layer in both c-GST and r-GST is absent in a-GST. This indicates the increased vacancy disorder. Thus, the combined structural and electronic information obtained here clearly suggests that the wavefunction localization in GST is driven by disorder of the vacancy distribution which increases the randomness of the vacancy clusters surrounding Te atoms. On re-crystallization, the vacancy layer reorders, thereby delocalizing the wave-functions, in accord with the electronic structure data discussed above. The results of this study therefore support the idea of vacancy order driven localization of the Te p-states as the origin of the metal-to-insulator transition in GST.

As the region near $\mathrm{E}_{F}$ is derived from $\mathrm{Te},{ }^{9,16}$ a detailed analysis of $\mathrm{Te} 3 \mathrm{~d}_{3 / 2}$ core level states was done to elucidate the role of vacancy co-ordination in changing the Te related electronic structure. The Te $3 \mathrm{~d}$ core-level spectra for all the three phases c-, a-, and r-GST could be uniquely fitted with two peaks having the same spectral width and a constant energy separation of $0.63 \mathrm{eV}$ (Fig. 5). The appearance of two peaks can be understood by the fact that Te has two environments: one being co-ordinated with $\mathrm{Ge}$ and $\mathrm{Sb}$ ions, while the second having co-ordination with the vacant site. A

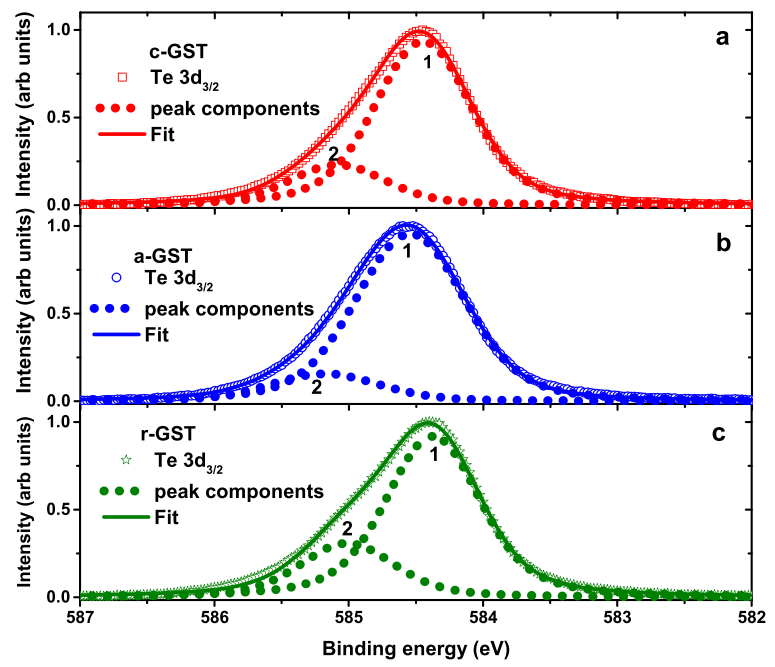

FIG. 5. Peak component analysis of Te $3 d_{3 / 2}$ states of (a) c-GST, (b) a-GST, and (c) r-GST. Peak 1 and Peak 2 corresponds to the non-vacancy and vacancy related components. vacant site co-ordination makes the Te-wavefunction more localized on Te sites. ${ }^{9}$ Due to a more bound electronic wavefunction for the vacancy co-ordinated $\mathrm{Te}$ atoms, the corresponding Te-3d peak component shifts to a higher binding energy (Peak 2, Fig. 5). The ratios between the area under the low-binding energy (peak 1, Fig. 5) and highbinding energy (peak 2, Fig. 5) of Te $3 d_{3 / 2}$ peak components for the c-, a-, and r-GST, are 4.07, 6.13, and 2.99, respectively. Clearly, for the insulating a-GST, the vacancy coordinated $\mathrm{Te} 3 \mathrm{~d}_{3 / 2}$ component (peak 2, Fig. 5) is weaker compared to that in the metallic crystalline (c- and r-) samples. This is consistent with the fact that there is a lack of vacancy ordering in the amorphous phase. This shows that the vacancy co-ordination modifies the electronic structure of $\mathrm{Te}$ states differently in metal and insulating states. Furthermore, it is found that the vacancy co-ordinated $\mathrm{Te}$ $3 \mathrm{~d}_{3 / 2}$ component is stronger in r-GST compared to that in the c-GST. This indicates that the observed difference in evolution of the electronic structure between c-GST and r-GST is possibly due to the enhanced vacancy interaction in the r-GST compared to that in the c-GST.

In conclusion, the combined spectroscopic and structural study on a reversibly switched crystalline thin film of a prototypical phase change material gives a clear and consistent picture of the electronic structure evolution among metallic, insulating, and re-crystallized metallic phases. The results clearly show the change in the initial state of Te as the transition from the crystalline metal to amorphous insulating state occurs. The high resolution diffraction data suggest that the change is associated with the change in vacancy order in the metallic and the insulating phases. The results resolve the ambiguity between previous spectroscopic data and the theoretical model of the origin of Anderson localization driven MIT in these phase change materials. Furthermore, it is found that on switching back from the amorphous (a-GST) to crystalline state (r-GST), the electronic structure near the fermi level of the final metallic state is different compared to the initial-metallic state from which the amorphous state is created. This point towards a different electronic structure evolution path for reversible switching from the metal to the insulator and back to the metal. This provides experimental information to advance the understanding of reversible electronic structure tuning in phase change materials. The results presented here should lead to further investigations for possible electronic structure changes with the change in various parameters like the film thickness and laser fluence.

See supplementary material for additional core level photoemission data and valence band spectra of r-GST for multiple crystallization cycles.

S.K. acknowledges financial support of the Department of Science and Technology (Government of India) provided within the framework of the India@DESY collaboration.

${ }^{1}$ D. Belitz and T. R. Kirkpatrick, Rev. Mod. Phys. 66, 261 (1994).

${ }^{2}$ M. Segev, Y. Silberberg, and D. N. Christodoulides, Nat. Photonics 7, 197 (2013).

${ }^{3}$ F. Evers and A. D. Mirlin, Rev. Mod. Phys. 80, 1355 (2008).

${ }^{4}$ K. Byczuk, W. Hofstetter, and D. Vollhardt, Int. J. Mod. Phys. B 24, 1727 (2010). 
${ }^{5}$ T. Siegrist, P. Jost, H. Volker, M. Woda, P. Merkelbach, C. Schlockermann, and M. Wuttig, Nat. Mater. 10, 202 (2011).

${ }^{6}$ M. Pasek, G. Orso, and D. Delande, Phys. Rev. Lett. 118, 170403 (2017).

${ }^{7}$ S. S. Kondov, W. R. McGehee, J. J. Zirbel, and B. DeMarco, Science 334, 66 (2011).

${ }^{8}$ G. Semeghini, M. Landini, P. Castilho, S. Roy, G. Spagnolli, A. Trenkwalder, M. Fattori, M. Inguscio1, and G. Modugno, Nat. Phys. 11, 554 (2015).

${ }^{9}$ W. Zhang, A. Thiess, P. Zalden, R. Zeller, P. H. Dederichs, J. Y. Raty, M. Wuttig, S. Blugel, and R. Mazzarello, Nat. Mater. 11, 952 (2012).

${ }^{10}$ A. Klein, H. Dieker, B. Spath, P. Fons, A. Kolobov, C. Steimer, and M. Wuttig, Phys. Rev. Lett. 100, 016402 (2008).

${ }^{11}$ X. Q. Liu, X. B. Li, L. Zhang, Y. Q. Cheng, Z. G. Yan, M. Xu, X. D. Han, S. B. Zhang, Z. Zhang, and E. Ma, Phys. Rev. Lett. 106, 025501 (2011).

${ }^{12}$ D. Campi, L. Paulatto, G. Fugallo, F. Mauri, and M. Bernasconi, Phys. Rev. B 95, 024311 (2017).

${ }^{13}$ V. Bragaglia, F. Arciprete, W. Zhang, A. M. Mio, E. Zallo, K. Perumal, A. Giussani, S. Cecchi, J. E. Boschker, H. Riechert, S. Privitera, E. Rimini, R. Mazzarello, and R. Calarco, Sci. Rep. 6, 23843 (2016).
${ }^{14}$ M. Wuttig and N. Yamada, Nat. Mater. 6, 824 (2007).

${ }^{15}$ Z. Sun, J. Zhou, and R. Ahuja, Phys. Rev. Lett. 96, 055507 (2006).

${ }^{16}$ T. Matsunaga, J. Akola, S. Kohara, T. Honma, K. Kobayashi, E. Ikenaga, R. O. Jones, N. Yamada, M. Takata, and R. Kojima, Nat. Mater. 10, 129 (2011).

${ }^{17}$ T. Kaewmaraya, M. Ramzan, H. Lofas, and R. Ahuja, J. Appl. Phys. 113, 033510 (2013).

${ }^{18}$ J. Akola, R. O. Jones, S. Kohara, S. Kimura, K. Kobayashi, M. Takata, T. Matsunaga, R. Kojima, and N. Yamada, Phys. Rev. B 80, 020201 (2009).

${ }^{19}$ T. Wei, J. Wei, K. Zhang, H. Zhao, and L. Zhang, Sci. Rep. 7, 42712 (2017).

${ }^{20}$ F. Jedema, Nat. Mater. 6, 90 (2007).

${ }^{21}$ J. J. Kim, K. Kobayashi, E. Ikenaga, M. Kobata, S. Ueda, T. Matsunaga, K. Kifune, R. Kojima, and N. Yamada, Phys. Rev. B 76, 115124 (2007).

${ }^{22}$ C. S. Fadley and D. A. Shirley, J. Res. Natl. Bur. Stand., Sect. A: Phys. Chem. 74, 543 (1970).

${ }^{23}$ A. Gloskovskii, G. Stryganyuk, G. H. Fecher, C. Felser, S. Thiess, H. Schulz-Ritter, W. Drube, G. Berner, M. Sing, R. Claessen, and M. Yamamoto, J. Electron Spectrosc. Relat. Phenom. 185, 47 (2012). 